\title{
CHEMICAL ENRICHMENT IN THE CARBON-ENHANCED DAMPED Ly $\alpha$ SYSTEM BY POPULATION III SUPERNOVAE
}

\author{
Chiaki Kobayashi ${ }^{1,2}$, Nozomu Tominaga ${ }^{2,3}$, and Ken'ICHi Nomoto ${ }^{2}$ \\ ${ }^{1}$ Research School of Astronomy \& Astrophysics, The Australian National University, Cotter Road, Weston, ACT 2611, Australia; chiaki@mso.anu.edu.au \\ 2 Institute for the Physics and Mathematics of the Universe, University of Tokyo, Kashiwa, Chiba 277-8583, Japan \\ ${ }^{3}$ Department of Physics, Faculty of Science and Engineering, Konan University, 8-9-1 Okamoto, Kobe, Hyogo 658-8501, Japan \\ Received 2010 December 14; accepted 2011 February 8; published 2011 March 3
}

\begin{abstract}
We show that the recently observed elemental abundance pattern of the carbon-rich metal-poor damped Ly $\alpha$ (DLA) system is in excellent agreement with the nucleosynthesis yields of faint core-collapse supernovae of primordial stars. The observed abundance pattern is not consistent with the nucleosynthesis yields of pair-instability supernovae. The DLA abundance pattern is very similar to that of carbon-rich extremely metal-poor (EMP) stars, and the contributions from low-mass stars and/or binary effects should be very small in DLAs. This suggests that chemical enrichment by the first stars in the first galaxies is driven by core-collapse supernovae from $\sim 20$ to $50 M_{\odot}$ stars and also supports the supernova scenario as the enrichment source of EMP stars in the Milky Way Galaxy.
\end{abstract}

Key words: galaxies: abundances - galaxies: evolution - quasars: absorption lines - stars: abundances - stars: Population III - supernovae: general

Online-only material: color figure

\section{INTRODUCTION}

At the end of the dark age of the universe, the cosmic dawn was heralded by the birth of the first stars and galaxies. The nature of these first objects is still far from being well understood. From the theory of star formation from primordial gas, the first stars are believed to be very massive, with masses of the order of $100 M_{\odot}$, given the limited cooling of molecular hydrogen (e.g., Bromm \& Larson 2004). This depends on fragmentation in a cosmological minihalo, ionization prior to the onset of gravitational collapse, and the accretion rate from the cloud envelope (Ohkubo et al. 2009, and references therein), and it seems possible to form lower-mass stars $\left(\sim 10-40 M_{\odot}\right)$ from primordial gas in recent numerical simulations (Yoshida et al. 2008; Stacy et al. 2009). The primordial stars with initial masses of $\sim 140-270 M_{\odot}$ explode as pair-instability supernovae (PISNe; Barkat et al. 1967; Umeda \& Nomoto 2002; Heger \& Woosley 2002). In terms of chemical abundances, however, no observational signature for the existence of PISNe has been detected.

Chemical enrichment from the first generation of stars has mainly been studied with extremely metal-poor (EMP) stars (e.g., Beers \& Christlieb 2005). Of EMP stars with [Fe/H] $\lesssim-2,10 \%-25 \%$ (Aoki 2010) show carbon enhancement relative to iron $([\mathrm{C} / \mathrm{Fe}] \gtrsim 1)$; this is also the case for the three hyper/ultra metal-poor stars known, with $[\mathrm{Fe} / \mathrm{H}]<-4.5$. Such stars are collectively known as carbon-enhanced metalpoor (CEMP) stars and are further subdivided as CEMP-s and CEMP-no stars depending on whether they exhibit or not an enhancement in the abundances of slow neutron capture elements such as barium. The abundance patterns of EMP stars are explained with one of, or the combination of, four enrichment sources: (1) core-collapse supernovae (Umeda \& Nomoto 2003; Iwamoto et al. 2005; Tominaga et al. 2007), (2) rotating massive stars (Meynet et al. 2006), (3) asymptotic giant branch (AGB) stars in binary systems (Suda et al. 2004), or (4) interstellar accretion (Yoshii 1981; Iben 1983). These enrichment sources have distinct signatures, so that it should be possible to distinguish between them by comparing the observed elemental abundances with theoretical calculations of nucleosynthetic yields.

The high $[\mathrm{C} / \mathrm{Fe}]$ can be explained with the mass transfer from AGB stars in binary systems, the mass loss from rotating massive stars, and a single core-collapse supernova forming a black hole. The Ba enhancement can be explained by AGB stars and possibly by rotating massive stars (Pignatari et al. 2008). However, iron-peak elements have to come from supernovae. The effect of interstellar accretion has not been studied with hydrodynamical simulations, but is observationally estimated to be negligible (Frebel et al. 2009). The binarity of CEMP stars has been studied by monitoring radial velocity variations. A signature of binarity is seen statistically for possibly all CEMP-s stars (Lucatello et al. 2005), but the binary fraction of CEMPno stars seems to be much lower (Aoki 2010). In particular, the binarity is not seen for the three stars with $[\mathrm{Fe} / \mathrm{H}]<-4.5$. Umeda \& Nomoto (2003) were the first to show that the observed abundance pattern from $\mathrm{C}$ to $\mathrm{Zn}$ can be well reproduced with the enrichment from a single core-collapse supernova that leaves behind a relatively massive black hole, under the assumption of inhomogeneous chemical enrichment (Audouse \& Silk 1995). Iwamoto et al. (2005) showed that the $\mathrm{N}$ abundance can be as large as observed with enhanced mixing between $\mathrm{H}$ and $\mathrm{He}$ layers during the hydrostatic stellar evolution.

The observations of very metal-poor damped Ly $\alpha$ (DLA) systems have opened a new window to study the chemical enrichment of the universe by the first generations of stars. DLAs are quasar absorbers defined by their high column density of neutral hydrogen, $\log N(\mathrm{HI}) / \mathrm{cm}^{-2} \geqslant 20.3$. They appear to sample a range of galaxy types, from the extended Hi disks of galaxies to smaller subgalactic size halos, as well as smaller H I clouds within larger galaxies (Wolfe et al. 2005). Largescale surveys, such as the Sloan Digital Sky Survey (SDSS), have increased 10-fold the number of known DLAs, which now number in excess of $\sim 1000$ (Noterdaeme et al. 2009; Prochaska $\&$ Wolfe 2009). Follow-up high-resolution spectroscopy of the most metal-poor DLAs is of particular interest, since the gas 
they trace may have been enriched by very few generations of stars (Pettini et al. 2008; Penprase et al. 2010). Moreover, measuring elemental abundances in DLAs is straightforward; the only potential complications are line saturation and dust depletion, and both effects are of much reduced importance for metallicities $Z \lesssim 1 / 100 Z_{\odot}$. In addition, the chemical evolution of such systems is rather simple, whereas in more chemically evolved systems, there are uncertainties in the star formation history, gas inflow and outflow, and large contributions from AGB stars and Type Ia supernovae, with the result that the signatures of the first stars can easily be washed out.

Thus, if the most metal-poor DLAs hold the key to unraveling the chemical enrichment from the first stars, it is of great interest to compare their observed abundance patterns with the nucleosynthesis yields of metal-free stars. Cooke et al. (2011) recently reported such a DLA with $[\mathrm{Fe} / \mathrm{H}] \simeq-3$, which exhibits a strong carbon enhancement relative to all other available elements, including $[\mathrm{C} / \mathrm{Fe}] \simeq+1.53$. This reminds us of the CEMP stars in the solar neighborhood. In this Letter, we compare the elemental abundance pattern of the C-rich DLA with the nucleosynthesis yields of both core-collapse and pairinstability supernovae (Section 2). In Section 3, we give a more general discussion of the chemical enrichment of the universe by the first generation of stars. We summarize our main conclusions in Section 4.

\section{ABUNDANCE PROFILING}

In stars with initial masses of $\gtrsim 10 M_{\odot}$, the iron core undergoes gravitational collapse at the end of the star's life. If sufficient amount of the released gravitational energy is transported to the outgoing shock wave, a supernova explosion occurs. The mechanism of core-collapse explosion and formation of a black hole remnant is still uncertain, although a few groups have presented feasible calculations of exploding supernovae (e.g., Marek \& Janka 2009). Thus, Umeda \& Nomoto (2002), Iwamoto et al. (2005), and Tominaga et al. (2007) have calculated nucleosynthesis yields by promptly exploding the progenitor model without calculating further collapse and bounce, and by applying mixing fallback. During the supernova explosion, the elements synthesized in different stellar layers mix and a large fraction of this mixed material falls back onto the remnant black hole. Physically, such a mixing-fallback process can be caused not only by the Rayleigh-Taylor instability (Hachisu et al. 1990; Joggerst et al. 2009) but also by a jet-induced explosion (Tominaga 2009). In the present study, we calculate nucleosynthesis yields with two new models of faint core-collapse supernovae (Nomoto et al. 2006) applying different mixingfallback parameters.

Figure 1 shows their elemental abundance ratios from $\mathrm{C}$ to $\mathrm{Zn}$ relative to $\mathrm{Fe}$. We adopt the progenitor star model from Iwamoto et al. (2005) for an initial mass of $M=25 M_{\odot}$ and metallicity $Z=0$. We implement two cases with different explosion energies: $1 \times 10^{51}$ erg (supernova $(\mathrm{SN})$, solid line) and $20 \times 10^{51}$ erg (hypernova (HN), short-dashed line). An efficient mixing fallback is adopted in both models: the mixing region of ejecta is assumed to be $M_{\text {in }}-M_{\text {out }}=1.76-6.14 M_{\odot}$ and 2.20-6.30 $M_{\odot}$ with the ejection fraction of $f=0.008$ and 0.004 , respectively. The resultant black hole masses are $6.1 M_{\odot}$ and $6.3 M_{\odot}$, respectively. The ejected Fe masses are as small as $M\left({ }^{56} \mathrm{Ni}\right)=M(\mathrm{Fe})=0.0018 M_{\odot}$ and $0.0014 M_{\odot}$, respectively, and thus correspond to faint supernovae such as SN1997D (Nomoto et al. 2006). For comparison, $M(\mathrm{Fe}) \simeq 0.07 M_{\odot}$ for normal supernovae and $\gtrsim 0.07 M_{\odot}$ for normal hypernovae

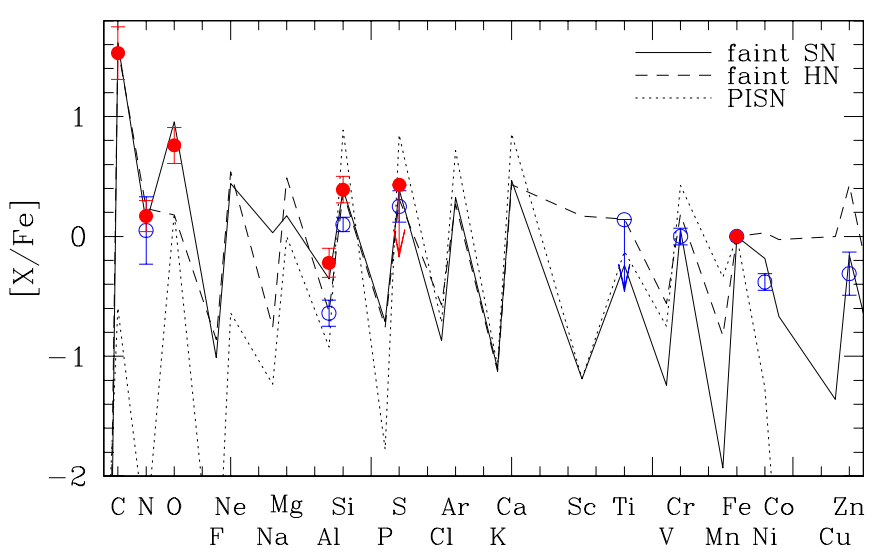

Figure 1. Elemental abundance pattern of the metal-poor C-rich DLA (filled circles) and peculiar DLA (open circles). The solid and short-dashed lines show the nucleosynthesis yields of faint core-collapse supernovae from $25 M_{\odot}$ stars with mixing fallback. The dotted line is for pair-instability supernovae from $170 M_{\odot}$ stars.

(A color version of this figure is available in the online journal.)

in Kobayashi et al. (2006, hereafter K06). The parameter dependencies of elemental abundance ratios can be summarized as follows.

1. The abundances of odd- $Z$ elements are lower than those of the $\alpha$ elements. This odd-even effect with the order of $\sim 0.5 \mathrm{dex}$ is realized for primordial supernovae in general and is consistent with observations of EMP stars in the Galactic halo (e.g., Cayrel et al. 2004). The yields of odd- $Z$ elements are enhanced by the surplus of neutrons in ${ }^{22} \mathrm{Ne}$, and ${ }^{22} \mathrm{Ne}$ is transformed from ${ }^{14} \mathrm{~N}$ during He burning. The absence of seed CNO elements in the progenitor stars results in smaller amounts of odd- $Z$ elements. This metallicity effect gives excellent agreement with the decreasing trends of $[(\mathrm{Na}, \mathrm{Al}, \mathrm{Cu}) / \mathrm{Fe}]$ toward lower metallicity in the solar neighborhood (K06). The metallicity effect is stronger for $\mathrm{Na}, \mathrm{Al}, \mathrm{Sc}$, and $\mathrm{Cu}$ than for $\mathrm{P}, \mathrm{Cl}, \mathrm{K}, \mathrm{V}, \mathrm{Mn}$, and $\mathrm{Co}$.

2. $\mathrm{C}$ and $\mathrm{N}$ are synthesized in the outermost region of the ejecta. Thus, the $[\mathrm{C} / \mathrm{Fe}]$ ratio is higher for smaller ejection of $\mathrm{Fe} .[\mathrm{N} / \mathrm{Fe}]$ depends on the initial metallicity and mixing during the stellar evolution.

3. $\alpha$ elements such as $\mathrm{O}, \mathrm{Mg}, \mathrm{Si}$, and $\mathrm{S}$ are synthesized in the outer regions of the ejecta. $[\mathrm{O} / \mathrm{Fe}]$ depends both on the explosion energy and mixing fallback, and thus it is not possible to put a constraint on the explosion energy only from $[\mathrm{O} / \mathrm{Fe}]$. Since $\mathrm{Mg}$ is more sensitive to the explosion energy, $[\mathrm{Mg} / \mathrm{Fe}]$ may give a clue to solve this degeneracy. $\mathrm{Si}$ and $\mathrm{S}$ are synthesized in more inner regions than $\mathrm{O}$, and significant amounts of $\mathrm{Si}$ and $\mathrm{S}$ fallback.

4. Zn, Co, and V are synthesized in the deep complete Siburning region together with $\sim 80 \%-90 \%$ of $\mathrm{Fe}$. [(Co, Zn)/ $\mathrm{Fe}] \gtrsim 0$ are realized in high-energy explosions with mixing fallback (Umeda \& Nomoto 2002); neutrino interactions could enhance [Zn/Fe] (Pruet et al. 2005; Fröhlich et al. 2006) but not [Co/Fe] (Izutani \& Umeda 2010).

5. $\mathrm{Cr}$ and $\mathrm{Mn}$ are mainly synthesized in the incomplete Siburning region. $[\mathrm{Cr} / \mathrm{Fe}]$ does not vary very much for these models; $\mathrm{Cr}$ abundance does not depend very much on parameters such as the electron fraction and entropy, and $[\mathrm{Cr} / \mathrm{Fe}]$ cannot be smaller with smaller $M_{\text {in }}$ or larger $M_{\text {out }}$. Larger $M_{\text {in }}$ can give larger $[\mathrm{Cr} / \mathrm{Fe}]$ and smaller [ $(\mathrm{Co}$, $\mathrm{Zn}) / \mathrm{Fe}$, but such an abundance pattern is not consistent with the EMP observations. Therefore, in galactic chemical 
evolution models, $[\mathrm{Cr} / \mathrm{Fe}]$ is almost constant over a wide range of $[\mathrm{Fe} / \mathrm{H}]$. This has been a problem when comparing with the observations (e.g., Cayrel et al. 2004), but now is consistent if NLTE effects are taken into account, as shown in K06.

We now compare these model calculations with the available abundance measurements in the extremely metal-poor DLA reported by Cooke et al. (2011, filled circles). This DLA was originally identified in the SDSS spectrum of the QSO J0035-0918. Follow-up high-resolution spectroscopy indicated $z_{\text {abs }}=2.3400972, \log N(\mathrm{HI}) / \mathrm{cm}^{-2}=20.55 \pm 0.1$, $[\mathrm{Fe} / \mathrm{H}] \simeq-3.04$, and a pronounced carbon enhancement $[\mathrm{C} / \mathrm{Fe}] \simeq+1.53$.

1. The observed $[\mathrm{C} / \mathrm{Fe}]$ ratio is well reproduced with our faint $\mathrm{SN} / \mathrm{HN}$ models. Low-mass AGB stars $\left(1-4 M_{\odot}\right)$ can also provide such high [C/Fe] (e.g., Karakas 2010). However, such low-mass stars are unlikely to contribute at the redshift of the C-rich DLA. With the star formation history in the solar neighborhood, $[\mathrm{C} / \mathrm{Fe}]$ reaches the maximum value at $z=1.8$ due to the AGB contribution (Kobayashi et al. 2011).

2. The $[\mathrm{O} / \mathrm{Fe}]$ ratio is higher than for non-faint supernovae $\left([\mathrm{O} / \mathrm{Fe}] \sim 0.5-0.6\right.$ for $25 M_{\odot}$ in $\left.\mathrm{K} 06\right)$ and is consistent with the faint $\mathrm{SN}$ model because of the smaller ejected $\mathrm{Fe}$ mass.

3. The $[(\mathrm{Si}, \mathrm{S}) / \mathrm{Fe}]$ ratios are similar to those of non-faint supernovae, and also consistent with the faint $\mathrm{SN} / \mathrm{HN}$ models.

4. The low Al abundance strongly suggests that the enrichment source is not Population II supernovae but primordial supernovae. Even for $Z=0.001,[\mathrm{Al} / \mathrm{Fe}]$ is larger than 0.35 for $\geqslant 20 M_{\odot}(\mathrm{K} 06)$.

5. The low $\mathrm{N}$ abundance is also consistent with the faint $\mathrm{SN} / \mathrm{HN}$ models, and the high $[\mathrm{C} / \mathrm{N}]$ ratio cannot be explained with mass loss from rotating massive stars (Meynet et al. 2010) or intermediate-mass AGB stars ( $\left.\gtrsim 4 M_{\odot}\right)$.

In order to discuss the detailed explosion mechanism of the supernova, it is necessary to obtain the elemental abundances of iron-peak elements. For the C-rich DLA, because of the low metallicity, it is impossible to detect heavier elements than $\mathrm{S}$ except for Fe. However, the abundance profiling approach can be used for other absorption systems with peculiar abundance patterns, because such systems have presumably been enriched by only a small number of supernovae. In Figure 1, we overplot (open circles) the element abundances recently reported by Cooke et al. (2010) for the $z_{\mathrm{abs}}=1.62650$ DLA in front of the gravitationally lensed quasar UM637A. This DLA, which has $\log N(\mathrm{HI}) / \mathrm{cm}^{-2}=20.7 \pm 0.1$ and $[\mathrm{Fe} / \mathrm{H}]=-1.56 \pm 0.03$, exhibits a different abundance pattern from other DLAs with similar metallicities and also from the average population of Galactic metal-poor stars. In particular, $\mathrm{Ti}, \mathrm{Ni}$, and $\mathrm{Zn}$ are deficient relative to $\mathrm{Fe}$ (see Figures 11 and 12 in Cooke et al. 2010).

We show that this abundance pattern can also be explained with faint supernovae. The normal $[(\mathrm{Si}, \mathrm{S}) / \mathrm{Fe}]$ ratios are consistent with the supernova scenario. The low $\mathrm{Al}$ abundance strongly suggests that the enrichment source is primordial supernovae. The observed $[\mathrm{Zn} / \mathrm{Fe}]$ ratio is more consistent with the faint $\mathrm{SN}$ model (solid line) than the faint $\mathrm{HN}$ model (dashed line) or non-faint supernovae without mixing fallback ([Zn/Fe] -1.7 in K06). This is also supported by the observed [Ni/Fe]. [Cr/Fe] is consistent with both supernova models. We should note that there is a problem in the Ti nucleosynthesis yields (K06), and we do not include $\mathrm{Ti}$ in the abundance profiling. At this metallicity, however, there is no information on $\mathrm{C}$ enhancement in the DLA observations since $C$ line is saturated. Therefore, it is uncertain how efficient the mixing fallback is. In fact, the observed abundances can be explained with medium mixing fallback of non-faint supernovae. In this case, the ejected Fe mass is larger, which may be consistent with the observed metallicity, and thus $[\mathrm{C} / \mathrm{Fe}]$ is lower than for the C-rich DLA.

In Figure 1, the dotted line is for the nucleosynthesis yields of the PISN of a $170 M_{\odot}$ star, which are taken from Umeda $\&$ Nomoto (2002). The primordial stars with initial masses of $\sim 140-270 M_{\odot}$ enter into the electron-positron pair-instability region during the central oxygen-burning stages and contract quasi-dynamically. Then the central temperature increases, central oxygen burning takes place explosively, and the generated nuclear energy is large enough to disrupt the stars completely without leaving compact remnants. Compared with corecollapse supernovae, the abundance pattern of PISNe can be summarized as follows.

1. The odd- $Z$ effect is much larger than $\sim 1$ dex.

2. $[(\mathrm{Si}, \mathrm{S}, \mathrm{Ar}, \mathrm{Ca}) / \mathrm{Fe}]$ are much larger than $[(\mathrm{O}, \mathrm{Mg}) / \mathrm{Fe}]$ because of more extensive explosive oxygen burning.

3 . $[\mathrm{Cr} / \mathrm{Fe}]$ is much larger because of the larger incomplete Si-burning region.

4. $[(\mathrm{Co}, \mathrm{Zn}) / \mathrm{Fe}]$ are much smaller because of the much larger ratio between the complete and incomplete Si-burning regions.

All of these characteristics disagree with the observed elemental abundances of these metal-poor DLAs. Even at high redshift, there is no signature of the existence of PISNe. The [Si/C] for PISNe is as large as +1.5 , which is also inconsistent with the observational estimate in the intergalactic medium (IGM) ([Si/C] 0.77; Aguirre et al. 2004). The IGM abundance looks more consistent with normal (non-faint) core-collapse supernovae with $[\mathrm{C} / \mathrm{Fe}] \sim 0$ and $[\mathrm{Si} / \mathrm{Fe}] \sim 0.7(\mathrm{~K} 06)$.

\section{DISCUSSION}

We have shown that the enrichment source of the extremely metal-poor DLA is very likely a primordial supernova that is faint as a result of mixing fallback to form a 3-6 $M_{\odot}$ black hole. It is interesting that the observed DLA abundance is very similar to those of EMP stars in the solar neighborhood including the ultra metal-poor star HE0557-4840 $([\mathrm{Fe} / \mathrm{H}]=-4.75$, $[\mathrm{C} / \mathrm{Fe}]=+1.6$; Norris et al. 2007) and BD+44 493 (Ito et al. 2009). Without rotation, the chemical enrichment from very massive stars $\left(\gtrsim 50-100 M_{\odot}\right)$ is very small because the central part is supposed to fall onto the black hole. In the early stages of galaxy formation, chemical enrichment is likely to be driven by core-collapse supernovae from $\sim 20$ to $50 M_{\odot}$ stars, although the mass range depends on the rotation.

How did the first supernova enrich the first galaxy? In our supernova scenario, we assume that the first supernova occurs in a primordial gas cloud with the total mass of $10^{6}-10^{7} M_{\odot}$. In hydrodynamical simulations (e.g., Mac Low \& Ferrara 1999), the interstellar medium (ISM) is ionized by the supernova explosion, and the H I mass rapidly decreases. Afterward, the H I mass slightly increases due to the recombination, and then decreases due to galactic mass outflow. Some of the ISM could have the $\mathrm{H}_{\mathrm{I}}$ column density of $\log N\left(\mathrm{H}_{\mathrm{I}}\right) \sim 20.5$ and could be observed as DLAs. The observable $\mathrm{H}$ I regions could have $M(\mathrm{HI}) \sim 3000 M_{\odot}$, although the HI mass highly depends 
on the total mass of the galaxy, the radial density profile, and the inhomogeneity of the ISM. With our faint supernova models, the ejected $\mathrm{C}$ mass is $\sim 0.2 M_{\odot}$, which is roughly consistent with the observed C-rich DLA. In Cooke et al. (2011), the masses of carbon and neutral gas of the C-rich DLA are estimated as $M(\mathrm{C} \mathrm{II}) \sim 2\left(n(\mathrm{H}) / 1 \mathrm{~cm}^{-3}\right)^{-2} M_{\odot}$ and $M(\mathrm{H} \mathrm{I}) \sim 2.5 \times 10^{4}\left(n(\mathrm{H}) / 1 \mathrm{~cm}^{-3}\right)^{-2} M_{\odot}$, which are consistent with our scenario if $n(\mathrm{H}) \sim 3 \mathrm{~cm}^{-3}$.

Are there any signatures of the first stars in other metal-poor systems? For globular cluster systems (GCSs), the present stellar mass and half-light radius are $10^{4}-10^{6} M_{\odot}$ and $1-35 \mathrm{pc}$ (e.g., Gilmore et al. 2007), respectively, which imply high densities $(n \sim 1000)$. Since the contribution of dark matter is small, the total mass would be $10^{5}-10^{9} M_{\odot}$ with the range of star formation efficiencies $(0.001-0.1)$. The progenitors of GCSs could be more massive if the GCS have lost a large fraction of stars by the relaxation as shown in $N$-body simulations (Lamers et al. 2010). Star formation takes place quickly, and $\gtrsim 10-1000$ supernovae are expected to occur from the stellar mass with the Salpeter initial mass function, which might blow away the ISM and quench star formation. This is consistent with the narrow metallicity distribution functions and the lack of the scatter in elemental abundance ratios (Carretta et al. 2009). In the elemental abundance patterns, no carbon enhancement that seems to be originated from supernovae is seen.

For dwarf spheroidal galaxies (dSphs), the present stellar mass and half-light radius are $10^{3}-10^{7} M_{\odot}$ and $20-1000 \mathrm{pc}$ (e.g., Gilmore et al. 2007), respectively, implying much lower densities than in GCSs. The dark matter mass is about $10^{7} M_{\odot}$ independent of the stellar mass (Geha et al. 2009). From the stellar mass, $1-10^{4}$ supernovae are expected. As seen in the observed color-magnitude diagrams (Tolstoy et al. 2009), star formation takes place slowly with a very low rate, and thus the ISM is likely to be inhomogeneous. The elemental abundance pattern can be used for the abundance profiling. In fact, a few EMP stars at $[\mathrm{Fe} / \mathrm{H}] \lesssim-3.5$ in $\mathrm{dSphs}$ do show carbon enhancement (Norris et al. 2010), which suggests a contribution from faint supernovae. Also in the outer halo of the Milky Way Galaxy, three stars at $[\mathrm{Fe} / \mathrm{H}] \lesssim-2.5$ do show $[\mathrm{C} / \mathrm{Fe}] \sim+2$ (T. C. Beers 2010, private communication); such stars may be disrupted from dSphs.

\section{CONCLUSIONS}

In the early stages of chemical enrichment, the ISM is supposed to be highly inhomogeneous, so that the properties of the first objects can be directly extracted from the comparison between the observed elemental abundances and nucleosynthesis yields. We have shown that the observed abundance pattern of the very metal-poor C-rich DLA is in excellent agreement with the nucleosynthesis yields of a primordial star that explodes as a faint core-collapse supernova owing to the efficient mixing and fallback. The nucleosynthesis yields of PISNe are not consistent with the observation. The contribution from rotating massive stars seems to be small because of the lack of $\mathrm{N}$ enhancement. The contribution from AGB stars should be very small because of the $\mathrm{N}$ abundance and of the enrichment timescale. Since the DLA abundances reflect the chemical enrichment in gas phase, the binary or accretion scenarios of the EMP stars do not work. Thus, we conclude that enrichment by primordial supernovae is the best solution to explain the abundance pattern of the C-rich DLA. The abundance pattern of the C-rich DLA is similar to those of EMP stars such as the ultra metal-poor star HE0557-4840. Some of EMP stars in
$\mathrm{dSphs}$ and the Galactic outer halo also show similar carbon enhancement at $[\mathrm{Fe} / \mathrm{H}] \lesssim-3$. Chemical enrichment by the first stars in the first galaxies is likely to be driven by core-collapse supernovae.

We thank M. Pettini and R. Cooke for providing their results prior to publication. We also thank J. Norris and R. Sutherland for fruitful discussion. This work has been supported in part by WPI Initiative, MEXT, Japan, and by the Grant-in-Aid for Scientific Research of the JSPS (18104003, 20540226) and MEXT (19047004, 22012003).

\section{REFERENCES}

Aguirre, A., Schaye, J., Kim, T.-S., Theuns, T., Rauch, M., \& Sargent, W. L. W. 2004, ApJ, 602, 38

Aoki, W. 2010, in IAU Symp. 265, Chemical Abundances in the Universe: Connecting First Stars to Planets, ed. K. Cunha et al. (Cambridge: Cambridge Univ. Press), 111

Audouse, J., \& Silk, J. 1995, ApJ, 451, L49

Barkat, Z., Rakavy, G., \& Sack, N. 1967, Phys. Rev. Lett., 18, 379

Beers, T. C., \& Christlieb, N. 2005, ARA\&A, 43, 531

Bromm, V., \& Larson, R. B. 2004, ARA\&A, 42, 79

Carretta, E., Bragaglia, A., Gratton, R., D’Orazi, V., \& Lucatello, S. 2009, A\&A, 508, 695

Cayrel, R., et al. 2004, A\&A, 416, 1117

Cooke, R., Pettini, M., Steidel, C. C., King, L. J., Rudie, G. C., \& Rakic, O. 2010, MNRAS, 409, 679

Cooke, R., Pettini, M., Steidel, C. C., Rudie, G. C., \& Jorgenson, R. A. 2011, MNRAS, in press

Frebel, A., Johnson, J. L., \& Bromm, V. 2009, MNRAS, 392, L50

Fröhlich, C., et al. 2006, ApJ, 637, 415

Geha, M., Willman, B., Simon, J. D., Strigari, L. E., Kirby, E. N., Law, D. R., \& Strader, J. 2009, ApJ, 692, 1464

Gilmore, G., Wilkinson, M. I., Wyse, R. F. G., Kleyna, J. T., Koch, A., Evans, N. W., \& Grebel, E. K. 2007, ApJ, 663, 948

Hachisu, I., Matsuda, T., Nomoto, K., \& Shigeyama, T. 1990, ApJ, 358, L57

Heger, A., \& Woosley, S. 2002, ApJ, 567, 532

Iben, I., Jr. 1983, Mem. Soc. Astron. Ital., 54, 321

Ito, H., Aoki, W., Honda, S., \& Beers, T. C. 2009, ApJ, 698, L37

Iwamoto, N, Umeda, H., Tominaga, N., Nomoto, K., \& Maeda, K. 2005, Science, 309,451

Izutani, N., \& Umeda, H. 2010, ApJ, 720, L1

Joggerst, C. C., Woosley, S. E., \& Heger, A. 2009, ApJ, 693, 1780

Karakas, A. I. 2010, MNRAS, 403, 1413

Kobayashi, C., Karakas, I. A., \& Umeda, H. 2011, MNRAS, in press

Kobayashi, C., Umeda, H., Nomoto, K., Tominaga, N., \& Ohkubo, T. 2006, ApJ, 653, 1145 (K06)

Lamers, H. J. G. L. M., Baumgardt, H., \& Girles, M. 2010, MNRAS, 409 , 305

Lucatello, S., Tsangarides, S., Beers, T. C., Carretta, E., Gratton, R. G., \& Ryan, S. G. 2005, ApJ, 625, 825

Mac Low, M., \& Ferrara, A. 1999, ApJ, 513, 142

Marek, A., \& Janka, H.-Th. 2009, ApJ, 694, 664

Meynet, G., Ekström, S., \& Maeder, A. 2006, A\&A, 447, 623

Meynet, G., Hirschi, R., Ekstrom, S., Maeder, A., Georgy, C., Eggenberger, P., \& Chiappini, C. 2010, A\&A, 521, 30

Nomoto, K., Tominaga, N., Umeda, H., Kobayashi, C., \& Maeda, K. 2006, Nucl. Phys. A, 777, 424

Norris, J. E., Christlieb, N., Korn, A. J., Eriksson, K., Bessell, M. S., Beers, T. C., Wisotzki, L., \& Reimers, D. 2007, ApJ, 670, 774

Norris, J. E., Gilmore, G., Wyse, R. F. G., Yong, D., \& Frebel, A. 2010, ApJ, 722,104

Noterdaeme, P., Petitjean, P., Ledoux, C., \& Srianand, R. 2009, A\&A, 505, 1087

Ohkubo, T., Nomoto, K., Umeda, H., Yoshida, N., \& Tsuruta, S. 2009, ApJ, 706, 1184

Penprase, B. E., Prochaska, J. X., Sargent, W. L. W., Toro Martinez, I., \& Beeler, D. J. 2010, ApJ, 721, 1

Pettini, M., Zych, B. J., Steidel, C. C., \& Chaffee, F. H. 2008, MNRAS, 385, 2011

Pignatari, M., Gallino, R., Meynet, G., Hirschi, R., Herwig, F., \& Wiescher, M. 2008, ApJ, 687, L95

Prochaska, J. X., \& Wolfe, A. M. 2009, ApJ, 696, 1543 
Pruet, J., Woosley, S. E., Buras, R., Janka, H.-T., \& Hoffman, R. D. 2005, ApJ, 623,325

Stacy, A. R., Greif, T. H., \& Bromm, V. 2009, MNRAS, 403, 45

Suda, T., Aikawa, M., Machida, M. N., Fujimoto, M. Y., \& Iben, I., Jr. 2004, ApJ, 611,476

Tolstoy, E., Hill, V., \& Tosi, M. 2009, ARA\&A, 47, 371

Tominaga, N. 2009, ApJ, 690, 526
Tominaga, N., Umeda, H., \& Nomoto, K. 2007, ApJ, 660, 516

Umeda, H., \& Nomoto, K. 2002, ApJ, 565, 385

Umeda, H., \& Nomoto, K. 2003, Nature, 422, 871

Wolfe, A. M., Gawiser, E., \& Prochaska, J. X. 2005, ARA\&A, 43, 861

Yoshida, N., Omukai, K., \& Hernquist, L. 2008, Science, 321, 669

Yoshii, Y. 1981, A\&A, 97, 280 